\title{
Full Averaging of Set Integrodifferential Equations
}

\author{
Tatyana A. Komleva ${ }^{1, *}$, Anastasiya V. Arsirii ${ }^{2}$ \\ ${ }^{1}$ Department Mathematics, Odessa State Academy Civil Engineering and Architecture, Odessa, 65029, Ukraine \\ ${ }^{2}$ Department Optimal Control, Odessa National University, Odessa, 65026, Ukraine
}

\begin{abstract}
In this article we prove the substantiation of the method of full averaging for the set integrodifferential equations with small parameter.
\end{abstract}

Keywords Set Integrodifferential Equation, Method of Averaging, Hukuhara Derivative

\section{Introduction}

As is generally known the absence of exact universal research methods for many important problems of analytical dynamics has caused the development of numerous approximate analytic and numerically-analytic methods that can be realized in effective computer algorithms.

The averaging methods combined with the asymptotic representations began to be applied as the basic constructive tool for solving the complicated problems of analytical dynamics described by the differential equations. It became possible due to the works of N.M. Krylov, N.N. Bogolyubov, Yu.A. Mitropolskij, V.M. Volosov, N.N. Moiseev, etc. (see [1-8]).

In recent years the development of the calculus in metric spaces has attracted some attention [6-12]. Earlier, F.S. de Blasi, F. Iervolino [13] started the investigation of set differential equations (SDEs) in semilinear metric spaces. This has now evolved into the theory of SDEs as an independent discipline: properties of solutions [6-45], the impulse equations [6,7,41], control systems [42-45] and asymptotic methods $[6-8,46-50]$. On the other hand, SDEs are useful in other areas of mathematics. For example, SDEs are used, as an auxiliary tool, to prove existence results for differential inclusions $[6,30,35,39]$. Also, one can employ SDEs in the investigation of fuzzy differential equations [7,10,25-27, 29,30]. Moreover, SDEs are a natural generalization of the usual ordinary differential equations in finite (or infinite) dimensional Banach spaces.

In this article we prove the substantiation of the method of full averaging for the set integrodifferential equations with small parameter. Thereby we expand a circle of systems to which it is possible to apply Krylov-Bogolyubov method of averaging.

* Corresponding author:

t-komleva@ukr.net (Tatyana A. Komleva)

Published online at http://journal.sapub.org/control

Copyright (C) 2011 Scientific \& Academic Publishing. All Rights Reserved

\section{Preliminaries}

Let $\operatorname{comp}\left(R^{n}\right)\left(\operatorname{conv}\left(R^{n}\right)\right)$ be a set of all nonempty (convex) compact subsets from the space $R^{n}$,

$$
h(A, B)=\min _{r \geq 0}\left\{S_{r}(A) \supset B, S_{r}(B) \supset A\right\}
$$

be Hausdorff distance between sets $A$ and $B, S_{r}(A)$ is $r$-neighborhood of set $A$.

Let $A, B, C$ be in $\operatorname{conv}\left(R^{n}\right)$. The set $C$ is the Hukuhara difference of $A$ and $B$, if $B+C=A$, i.e.

$$
C=A \frac{H}{B} \text {. }
$$

From Radstrom's Cancellation Lemma [51], it follows that if this difference exists, then it is unique.

Definition [52]. A mapping $X:[0, T] \rightarrow \operatorname{conv}\left(R^{n}\right)$ is differentiable in the sense of Hukuhara at $t \in[0, T]$ if for some $\delta>0$ the Hukuhara differences

$$
X(t+\Delta) \frac{H}{X} X(t), \quad X(t) \frac{H}{X} X(t-\Delta)
$$

exists in $\operatorname{conv}\left(R^{n}\right)$ for all $0<\Delta<\delta$ and there exists an $D X(t) \in \operatorname{conv}\left(R^{n}\right)$ such that

$$
\lim _{\Delta \rightarrow 0_{+}} h\left(\Delta^{-1}\left(X(t+\Delta) \frac{H}{X} X(t)\right), D X(t)\right)=0
$$

and

$$
\lim _{\Delta \rightarrow 0_{+}} h\left(\Delta^{-1}\left(X(t) \frac{H}{X} X(t-\Delta)\right), D X(t)\right)=0 .
$$

Here $D X(t)$ is called the Hukuhara derivative of $X(t)$ at $t$.

\section{The Scheme of Full Average}

Consider the Cauchy problem with small parameter 


$$
D X=\varepsilon F\left(t, X, \int_{0}^{t} \Phi(t, s, X(s)) d s\right), \quad X(0)=X_{0},
$$

where $\varepsilon>0$ is a small parameter, $t, s \in R_{+}$, $F: R_{+} \times \operatorname{conv}\left(R^{n}\right) \times \operatorname{conv}\left(R^{m}\right) \rightarrow \operatorname{conv}\left(R^{n}\right)$ is a set mapping, $\Phi: R_{+} \times R_{+} \times \operatorname{conv}\left(R^{n}\right) \rightarrow \operatorname{comp}\left(R^{m}\right)$ is a set mapping, Here the integral is understood in the sense of [52] (the integral exists for example if $X(\cdot)$ is measurable and the real mapping $t \rightarrow h(X(t),\{0\})$ is integrable on $\left.I \subset R_{+}\right)$, $X_{0} \in \operatorname{conv}\left(R^{n}\right)$.

Definition. A mapping $X:[0, T] \rightarrow \operatorname{conv}\left(R^{n}\right)$ is a solution to the problem (1) if and only if it is continuous and satisfies the integral equation

$$
X(t)=X_{0}+\varepsilon \int_{0}^{t} F\left(\tau, X(\tau), \int_{0}^{\tau} \Phi(\tau, s, X(s)) d s\right) d \tau
$$

for all $t \in[0, T]$.

In the beginning this section we associate with the equation (1) the following full averaged integrodifferential equation

$$
D Y=\varepsilon \bar{F}(Y), \quad Y(0)=X_{0},
$$

where

$$
\begin{gathered}
\lim _{T \rightarrow \infty} h\left(\frac{1}{T} \int_{0}^{T} F_{1}(t, X) d t, \bar{F}(X)\right)=0, \\
F_{1}(t, X) \equiv F(t, X, \Psi(t, X)), \Psi(t, X) \equiv \int_{0}^{t} \Phi(t, s, X) d s
\end{gathered}
$$

Remark. In the beginning we will consider a case when the limit (3) exists.

Definition. We say that the limit (3) exists uniformly in $X$, if for any $\alpha>0$ and any $X \in G \in \operatorname{conv}\left(R^{n}\right)$ there exists $T(\alpha)$ such that.

$$
h\left(\frac{1}{T} \int_{0}^{T} F_{1}(t, X) d t, \bar{F}(X)\right)<\alpha,
$$

for all $T>T(\alpha)$.

Theorem. Let in domain.

$$
Q=\left\{(t, X) \mid t \geq 0, X \in G \in \operatorname{conv}\left(R^{n}\right)\right\}
$$

the following hold:

1) $F(t, X, Z)$ is continuous in

$$
(t, X, Z) \in R_{+} \times G \times \operatorname{conv}\left(R^{m}\right) ;
$$

2) $\Phi(t, s, X)$ is continuous in $(t, s, X) \in R_{+} \times R_{+} \times G$;

3 ) there exist continuous function $\mu(t, s)$, and constant $\lambda$ such that

$$
\begin{gathered}
h\left(F\left(t, X_{1}, Z_{1}\right), F\left(t, X_{2}, Z_{2}\right)\right) \leq \lambda\left[h\left(X_{1}, X_{2}\right)+h\left(Z_{1}, Z_{2}\right)\right], \\
h\left(\Phi\left(t, s, X_{1}\right), \Phi\left(t, s, X_{2}\right)\right) \leq \mu(t, s) h\left(X_{1}, X_{2}\right),
\end{gathered}
$$

for any $X_{1}, X_{2} \in G, Z_{1}, Z_{2} \in \operatorname{conv}\left(R^{m}\right)$;

4) $\lim _{t \rightarrow \infty} \bar{\mu}_{0}(t)=0$, where

$$
\bar{\mu}_{0}(t) \equiv \frac{1}{t} \int_{0}^{t} \mu_{0}(\tau) d \tau, \mu_{0}(\tau) \equiv \int_{0}^{\tau} \mu(\tau, s) d s
$$

5) there exist constants $v, M$ such that

$$
h\left(\bar{F}\left(X_{1}\right), \bar{F}\left(X_{2}\right)\right) \leq \nu h\left(X_{1}, X_{2}\right), h(\bar{F}(X),\{0\}) \leq M,
$$

for any $X, X_{1}, X_{2} \in G$;

6) the limit (3) exist uniformly in $X \in G$;

7) for any $X_{0} \in G^{\prime} \subset G$ and $t \geq 0$ the solution of the equation (2) together with a $\sigma$-neighborhood belong to the domain $G$.

Then for any $\eta>0$ and $L>0$ there exists $\varepsilon^{0}(\eta, L) \in(0, \sigma]$ such that for all $\varepsilon \in\left(0, \varepsilon^{0}\right]$ and $t \in\left[0, L \varepsilon^{-1}\right]$ the following statement fulfill:

$$
h(X(t), Y(t))<\eta,
$$

where $X(t), Y(t)$ are the solutions of the initial and the full averaged equations.

Proof. Since.

$$
\begin{gathered}
X(t)=X_{0}+\varepsilon \int_{0}^{t} F\left(\tau, X(\tau), \int_{0}^{\tau} \Phi(\tau, s, X(s)) d s\right) d \tau, \\
Y(t)=X_{0}+\varepsilon \int_{0}^{t} \bar{F}(Y(\tau)) d \tau,
\end{gathered}
$$

we have $h(X(t), Y(t)) \leq$

$$
\begin{gathered}
\leq \varepsilon h\left(\int_{0}^{t} F\left(\tau, X(\tau), \int_{0}^{\tau} \Phi(\tau, s, X(s)) d s\right) d \tau,\right. \\
\left.\quad \int_{0}^{t} F\left(\tau, Y(\tau), \int_{0}^{\tau} \Phi(\tau, s, Y(s)) d s\right) d \tau\right)+ \\
+\varepsilon h\left(\int_{0}^{t} F\left(\tau, Y(\tau), \int_{0}^{\tau} \Phi(\tau, s, Y(s)) d s\right) d \tau,\right. \\
+\varepsilon h\left(\int_{0}^{t} F\left(\tau, Y(\tau), \int_{0}^{t} \Phi\left(\tau, Y(\tau), \int_{0}^{\tau} \Phi(\tau, s, Y(\tau)) d s\right) d \tau\right)+\right.
\end{gathered}
$$

Hence $h(X(t), Y(t)) \leq$

$$
\leq \varepsilon \int_{0}^{t} \lambda\left[h(X(\tau), Y(\tau))+h\left(\int_{0}^{\tau} \Phi(\tau, s, X(s)) d s, \int_{0}^{\tau} \Phi(\tau, s, Y(s)) d s\right)\right] d \tau+
$$$$
+\varepsilon \int_{0}^{t} \lambda h\left(\int_{0}^{\tau} \Phi(\tau, s, Y(s)) d s, \int_{0}^{\tau} \Phi(\tau, s, Y(\tau)) d s\right) d \tau+
$$$$
+\varepsilon h\left(\int_{0}^{t} F\left(\tau, Y(\tau), \int_{0}^{\tau} \Phi(\tau, s, Y(\tau)) d s\right) d \tau, \int_{0}^{t} \bar{F}(Y(\tau)) d \tau\right) .
$$

Then we obtain $h(X(t), Y(t)) \leq$ 


$$
\begin{aligned}
& \leq \varepsilon \lambda \int_{0}^{t} h(X(\tau), Y(\tau)) d \tau+\varepsilon \lambda \int_{0}^{t} \int_{0}^{\tau} \mu(\tau, s) h(X(s), Y(s)) d s d \tau+ \\
& +\varepsilon \lambda \int_{0}^{t} \int_{0}^{\tau} \mu(\tau, s) h(Y(s), Y(\tau)) d s d \tau+ \\
& +\varepsilon h\left(\int_{0}^{t} F\left(\tau, Y(\tau), \int_{0}^{\tau} \Phi(\tau, s, Y(\tau)) d s\right) d \tau, \int_{0}^{t} \bar{F}(Y(\tau)) d \tau\right)(6)
\end{aligned}
$$

In the beginning we will estimate last summand in (6). Divide the interval $\left[0, L \varepsilon^{-1}\right]$ into partial intervals by the points $t_{i}=\frac{i L}{m \varepsilon}, \quad i=0, \ldots, m, m \in \mathrm{N}$.

Then

$$
\begin{gathered}
\varepsilon h\left(\int_{0}^{t} F\left(\tau, Y(\tau), \int_{0}^{\tau} \Phi(\tau, s, Y(\tau)) d s\right) d \tau, \int_{0}^{t} \bar{F}(Y(\tau)) d \tau\right) \leq \\
\leq \Sigma_{1}+\Sigma_{2}+\Sigma_{3},
\end{gathered}
$$

where

$$
\begin{gathered}
\Sigma_{1}=\varepsilon \sum_{i=0}^{m-1} \int_{t_{i}}^{t_{i+1}} h\left(F\left(\tau, Y(\tau), \int_{0}^{\tau} \Phi(\tau, s, Y(\tau) d s)\right),\right. \\
\left.\Sigma_{2}=\varepsilon \sum_{i=0}^{m-1} \int_{t_{i}}^{t_{i+1}} h\left(\tau, Y\left(t_{i}\right), \int_{0}^{\tau} \Phi\left(\tau, s, Y\left(t_{i}\right)\right) d s\right)\right) d \tau, \\
\Sigma_{3}=\varepsilon \sum_{i=0}^{m-1} h\left(\int_{t_{i}}^{t_{i+1}} F\left(\tau, Y\left(t_{i}\right)\right), \int_{0}^{\tau} \Phi\left(\tau, s, Y\left(t_{i}\right)\right) d s\right) d \tau, \\
\left.\int_{t_{i}}^{t_{i+1}} \bar{F}\left(Y\left(t_{i}\right)\right) d \tau\right) .
\end{gathered}
$$

As $h\left(Y(\tau), Y\left(t_{i}\right)\right) \leq \varepsilon M\left|\tau-t_{i}\right|$ for all $\tau \in\left[t_{i}, t_{i+1}\right]$, $i=\overline{0, m-1}$; then $\Sigma_{1} \leq$

$$
\begin{gathered}
\leq \varepsilon \sum_{i=0}^{m-1_{t_{i}}^{t_{i+1}}} \int_{t_{i}}\left[\lambda h\left(Y(\tau), Y\left(t_{i}\right)\right)+\lambda \int_{0}^{\tau} \mu(\tau, s) h\left(Y(\tau), Y\left(t_{i}\right)\right) d s\right] d \tau \leq \\
\leq \varepsilon^{2} \lambda M \sum_{i=0}^{m-1} \int_{t_{i}}^{t_{i+1}}\left[\left(\tau-t_{i}\right)+\mu_{0}(\tau)\left(\tau-t_{i}\right)\right] d \tau \leq \\
\leq \lambda \frac{M L^{2}}{2 m}+\varepsilon \lambda \frac{M L}{m} \int_{0}^{L / \varepsilon} \mu_{0}(\tau) d \tau \\
\Sigma_{2} \leq \varepsilon \nu \sum_{i=0}^{m-1} \int_{t_{i}}^{t_{i+1}} h\left(Y(\tau), Y\left(t_{i}\right)\right) d \tau \leq \varepsilon^{2} \nu M \sum_{i=0}^{m-1} \int_{t_{i}}^{t_{i+1}}\left(\tau-t_{i}\right) d \tau \leq \\
\leq \frac{\nu M L^{2}}{2 m} .
\end{gathered}
$$

Hence

$$
\begin{gathered}
\Sigma_{1}+\Sigma_{2} \leq \frac{M L^{2}}{2 m}(\lambda+\nu)+\varepsilon \frac{\lambda M L}{m} \int_{0}^{L / \varepsilon} \mu_{0}(\tau) d \tau \leq \\
\leq \frac{M L^{2}}{2 m}(\lambda+\nu)+\frac{\lambda M L}{m} \beta(\varepsilon) \equiv \alpha(m, \varepsilon),
\end{gathered}
$$

where $\beta(\varepsilon) \equiv \sup _{0 \leq \tau \leq L} \tau \bar{\mu}_{0}\left(\frac{\tau}{\varepsilon}\right) \geq \varepsilon t \bar{\mu}_{0}(t)=\varepsilon \int_{0}^{t} \mu_{0}(\tau) d \tau$.

Obviously, $\lim _{m \rightarrow \infty} \alpha(m, \varepsilon)=0$, and $\lim _{\varepsilon \rightarrow 0} \beta(\varepsilon)=0$.

Now, we will estimate $\Sigma_{3}$. From condition 6) of the theorem it follows that there exists the increasing function $\theta(t)$ such that

1) $\lim _{t \rightarrow \infty} \theta(t)=0$;

2) $h\left(\int_{0}^{t} F_{1}(\tau, X), d \tau, \int_{0}^{t} \bar{F}(X) d \tau\right) \leq t \theta(t)$.

Then $\Sigma_{3} \leq$

$$
\begin{gathered}
\leq \varepsilon \sum_{i=0}^{m-1} h\left(\int_{0}^{t_{i+1}} F\left(\tau, Y\left(t_{i}\right), \int_{0}^{\tau} \Phi\left(\tau, s, Y\left(t_{i}\right)\right) d s\right) d \tau, \int_{0}^{t_{i+1}} \bar{F}\left(Y\left(t_{i}\right)\right) d \tau\right)+ \\
+\varepsilon \sum_{i=0}^{m-1} h\left(\int_{0}^{t_{i}} F\left(\tau, Y\left(t_{i}\right), \int_{0}^{\tau} \Phi\left(\tau, s, Y\left(t_{i}\right)\right) d s\right) d \tau, \int_{0}^{t_{i}} \bar{F}\left(Y\left(t_{i}\right)\right) d \tau\right) \leq \\
\leq 2 m \psi(\varepsilon),
\end{gathered}
$$

where $\psi(\varepsilon)=\sup _{\tau \in[0, L]}\left(\tau \theta\left(\frac{\tau}{\varepsilon}\right)\right), \quad \tau=\varepsilon t$.

By (6), we have $h(X(t), Y(t)) \leq$

$$
\begin{gathered}
\leq \varepsilon \lambda \int_{0}^{t} h(X(\tau), Y(\tau)) d \tau+\varepsilon \lambda \int_{0}^{t} \int_{0}^{\tau} \mu(\tau, s) h(X(s), Y(s)) d s d \tau+ \\
+\varepsilon \lambda \int_{0}^{t} \int_{0}^{\tau} \mu(\tau, s) h(Y(s), Y(\tau)) d s d \tau+\alpha(m, \varepsilon)+2 m \psi(\varepsilon) \leq \\
\leq \varepsilon \lambda \int_{0}^{t} h(X(\tau), Y(\tau)) d \tau+\varepsilon \lambda \int_{0}^{t} \int_{0}^{\tau} \mu(\tau, s) h(X(s), Y(s)) d s d \tau+ \\
+\lambda M L \beta(\varepsilon)+\alpha(m, \varepsilon)+2 m \psi(\varepsilon) .
\end{gathered}
$$

Using Gronwall-Bellman's inequality, we obtain $h(X(t), Y(t)) \leq(\alpha(m, \varepsilon)+\lambda M L \beta(\varepsilon)+2 m \psi(\varepsilon)) e^{\lambda L+\lambda \beta(\varepsilon)}$

Fix $m$. Then for any $\eta>0$, there exists $\varepsilon_{0}>0$ such that the following estimate is true for $0<\varepsilon<\varepsilon_{0}$ :

$$
\begin{gathered}
\alpha(m, \varepsilon)+\lambda M L \beta(\varepsilon)+2 m \psi(\varepsilon)<\min \{\eta, \sigma\} e^{-\lambda(1+L)}, \\
\beta(\varepsilon)<1 .
\end{gathered}
$$

Hence we obtain $h(X(t), Y(t)) \leq \eta$ for all $t \in\left[0, L \varepsilon^{-1}\right]$.

This concludes the proof.

Now we consider a case when the limit (3) not exists. Then we associate with the equation (1) the following integrodifferential equation

$$
D Y=\varepsilon F\left(t, Y, \int_{0}^{t} \Phi(t, s, Y(t)) d s\right), \quad Y(0)=X_{0} .
$$


Theorem. Let in domain

$$
Q=\left\{(t, X) \mid t \geq 0, X \in G \in \operatorname{conv}\left(R^{n}\right)\right\}
$$

the following hold:

1) $F(t, X, Z)$ is continuous in

$$
(t, X, Z) \in R_{+} \times G \times \operatorname{conv}\left(R^{m}\right) ;
$$

2) $\Phi(t, s, X)$ is continuous in $(t, s, X) \in R_{+} \times R_{+} \times G$;

3) there exist continuous function $\mu(t, s)$, and constants $\lambda \quad M$ such that

$$
\begin{gathered}
h\left(F\left(t, X_{1}, Z_{1}\right), F\left(t, X_{2}, Z_{2}\right)\right) \leq \lambda\left[h\left(X_{1}, X_{2}\right)+h\left(Z_{1}, Z_{2}\right)\right], \\
h\left(\Phi\left(t, s, X_{1}\right), \Phi\left(t, s, X_{2}\right)\right) \leq \mu(t, s) h\left(X_{1}, X_{2}\right), \\
h(F(t, X, Z),\{0\}) \leq M,
\end{gathered}
$$

for any $X, X_{1}, X_{2} \in G, Z, Z_{1}, Z_{2} \in \operatorname{conv}\left(R^{m}\right)$;

4) $\lim _{t \rightarrow \infty} \bar{\mu}_{0}(t)=0$, where

$$
\bar{\mu}_{0}(t) \equiv \frac{1}{t} \int_{0}^{t} \mu_{0}(\tau) d \tau, \mu_{0}(\tau) \equiv \int_{0}^{\tau} \mu(\tau, s) d s ;
$$

5) for any $X_{0} \in G^{\prime} \subset G$ and $t \geq 0$ the solutions of the equations (1) and (7) together with a $\sigma$-neighborhood belong to the domain $G$.

Then the statement (8) fulfill:

$$
h(X(t), Y(t))<\lambda M L \beta(\varepsilon) e^{\lambda L+\lambda \beta(\varepsilon)},
$$

where $t \in\left[0, L \varepsilon^{-1}\right], \beta(\varepsilon)=\sup _{0 \leq \tau \leq L} \tau \bar{\mu}_{0}\left(\frac{\tau}{\varepsilon}\right)$.

Proof. By (1) and (7) we have $h(X(t), Y(t)) \leq$

$$
\begin{gathered}
\leq \operatorname{sh}\left(\int_{0}^{t} F\left(\tau, X(\tau), \int_{0}^{\tau} \Phi(\tau, s, X(s)) d s\right) d \tau,\right. \\
\left.\quad \int_{0}^{t} F\left(\tau, Y(\tau), \int_{0}^{\tau} \Phi(\tau, s, Y(s)) d s\right) d \tau\right)+ \\
+\operatorname{\varepsilon h}\left(\int_{0}^{t} F\left(\tau, Y(\tau), \int_{0}^{\tau} \Phi(\tau, s, Y(s)) d s\right) d \tau,\right. \\
\left.\int_{0}^{t} F\left(\tau, Y(\tau), \int_{0}^{\tau} \Phi(\tau, s, Y(\tau)) d s\right) d \tau\right) .
\end{gathered}
$$

Hence we obtain $h(X(t), Y(t)) \leq$

$$
\begin{gathered}
\leq \varepsilon \lambda \int_{0}^{t} h(X(\tau), Y(\tau)) d \tau+\varepsilon \lambda \int_{0}^{t} \int_{0}^{\tau} \mu(\tau, s) h(X(s), Y(s)) d s d \tau+ \\
+\varepsilon \lambda \int_{0}^{t} \int_{0}^{\tau} \mu(\tau, s) h(Y(s), Y(\tau)) d s d \tau
\end{gathered}
$$

As we have for all $t \in\left[0, L \varepsilon^{-1}\right]$

$$
h(Y(s), Y(\tau)) \leq \varepsilon M|s-\tau| \leq M L ;
$$

then by (9) we obtain (8). This concludes the proof.

\section{Conclusions}

Here we used the approach of Hukuhara at definition of the derivative which has essential shortages. However the given approach is well investigated by many authors. Also in the literature exist other approaches to definition of the derivative $[7,8,11,12,25,40]$, but also they have the shortages. It is easily possible to show that this outcome will be true for some other cases with little changes.

\section{REFERENCES}

[1] N. M. Krylov and N. N. Bogoliubov, Introduction to nonlinear mechanics. Princeton, Princeton University Press, 1947

[2] N. N. Bogoliubov and Yu. A. Mitropolsky, Asymptotic methods in the theory of non-linear oscillations. New York, Gordon and Breach, 1961

[3] J. A. Sanders and F. Verhulst, Averaging methods in nonlinear dynamical systems. Appl. Math. Sci., Springer-Verlag, New York, 1985, vol. 59

[4] V. M. Volosov and B. I. Morgunov, Metod of average in the theory of nonlinear oscillations. Moscow, Moscow State University Publishing house, 1971

[5] P. Lochak, C. Meunier, Multiphase averaging for classical systems. Appl. Math. Sci., Springer-Verlag, New York, 1988, vol. 72

[6] N. A. Perestyuk, V. A. Plotnikov, A. M. Samoilenko and N. V. Skripnik, Differential equations with impulse effects: multivalued right-hand sides with discontinuities. De Gruyter Studies in Mathematics. Berlin/Boston: Walter De Gruyter GmbH\&Co., 2011, vol. 40

[7] A. V. Plotnikov and N. V. Skripnik, Differential equations with "clear" and fuzzy multivalued right-hand sides. Asymptotics Methods. AstroPrint, Odessa, 2009

[8] V. A. Plotnikov, A. V. Plotnikov and A. N. Vityuk, Differential equations with a multivalued right-hand side: Asymptotic methods. AstroPrint, Odessa, 1999

[9] V. Lakshmikantham, T. Granna Bhaskar and J. Vasundhara Devi, Theory of set differential equations in metric spaces. Cambridge Scientific Publishers. 2006

[10] V. Lakshmikantham and R. N. Mohapatra, Theory of Fuzzy Differential Equations and Inclusions. London, Taylor \& Francis, 2003

[11] Plotnikov, A.V. and Skripnik, N.V., 2011, Set-valued differential equations with generalized derivative., J. Advanced Research in Pure Mathematics, 13(1), 144-160

[12] de Blasi, F. S. and Iervolino, F., 1969, Equazioni differentiali con soluzioni a valore compatto convesso., Boll. Unione Mat. Ital., 2(4-5), 491-501

[13] Bede, B. and Stefanini, L., 2008, Generalized Hukuhara differentiability of interval-valued functions and interval differential equations., Univ. Urbino "Carlo Bo", Working Paper Series in Economics, Math. and Statistics. WPEMS\#2008/03 
[14] de Blasi, F. S. and Iervolino, F., 1971, Euler method for differential equations with set-valued solutions., Boll. Unione Mat. Ital., 4(4), 941-949

[15] Brandao Lopes Pinto, A. J., de Blasi, F. S. and Iervolino, F., 1970, Uniqueness and existence theorems for differential equations with compact convex valued solutions., Boll. Unione Mat. Ital., (4), 534-538

[16] Dabrowska, R.. and Janiak, T., 1993, Stability of functionaldifferential equations with compact convex valued solutions., Discuss. Math., (13), 87-92

[17] Drici, Z., Mcrae, F. A. and Vasundhara Devi, J., 2005, Set differential equations with causal operators., Mathematical Problems in Engineering, 2005:2, 185-194

[18] Galanis, G. N., Gnana Bhaskar, T., Lakshmikantham, V., and Palamides, P. K., 2005, Set value functions in Frechet spaces: Continuity, Hukuhara differentiability and applications to set differential equations., J. Nonlinear Analysis, 61, $559-575$

[19] Galanis, G. N., Tenali, G. B. and Lakshmikantham, V., 2008, Set differential equations in Frechet spaces., J. Appl. Anal., $14,103-113$

[20] Gnana Bhaskar, T. and Lakshmikantham, V., 2003, Set differential equations and flow invariance., Appl. Anal. (82), 357-368

[21] Gnana Bhaskar, T. and Lakshmikantham, V., 2004, Lyapunov stability for set differential equations., Dynam. Systems Appl., (13), 1-10

[22] Gnana Blaskar, T. and Vasundhara Devi, J., 2005, Set differential systems and vector Lyapunov functions., Appl. Math. Comput., 165(3), 539-548.

[23] Kisielewicz, M., 1975, Description of a class of differential equations with set-valued solutions., Lincei-Rend. Sc. fis. mat. e nat., 58, 158-162

[24] Kisielewicz, M., Serafin, B. and Sosulski, W., 1975, Existence theorem for functional-differential equation with compact convex valued solutions., Demonstratio math., 13(2), 229-237

[25] Komleva, T. A., Plotnikov, A. V. and Skripnik, N. V., 2008, Differential equations with set-valued solutions., Ukr. Math. J., 60(10), 1540-1556

[26] Lakshmikantham, V., 2004, The connection between set and fuzzy differential equations., Facta Univ. Ser. Mech. Automat. Control. Robot., 4, 1-10

[27] Lakshmikantham, V., 2005, Set differential equations versus fuzzy differential equations., J. Applied Mathematics and Computation, 164, 277-294

[28] Laksmikantham, V., Leela, S. and Vatsala, A. S., 2002, Setvalued hybrid differential equations and stability in terms of two measures., J. Hybrid Systems, (2), 169-187

[29] Laksmikantham, V., Leela, S. and Vatsala, A. S., 2003, Interconnection between set and fuzzy differential equations., Nonlinear Anal., 54, 351-360

[30] Lakshmikantham, V. and Tolstonogov, A. A., 2003, Existence and interrelation between set and fuzzy differential equations., Nonlinear Anal., 55, 255-268
[31] Piszczek, M., 2006, Second Hukuhara derivative and cosine family of linear set-valued functions., An. Acad. Paedagogicae Cracoviensis. Studia Math., 33, 87-98

[32] Piszczek, M., 2008, On a multivalued second order differential problem with Hukuhara derivative., Opuscula Math., 28(2), 151-161

[33] Plotnikov, A. V., 2000, Differentiation of multivalued mappings. T-derivative., Ukr. Math. J., 52(8), 1282-1291

[34] Plotnikov, A. V. and Tumbrukaki, A. V., 2000, Integro-differential equations with multivalued solutions., Ukr. Math. J., 52(3), 413-423

[35] Plotnikova, N. V., 2006, Approximation of a set of solutions of linear differential inclusions., Nonlinear Oscil., 9(3), 375-390

[36] Skripnik, N. V., 2008, The Krasnoselskii-Krein theorem for differential equations with multivalued solutions., Visn. Khark. Univ., Ser. Mat. Prykl. Mat. Mekh., 826(58), 87-99

[37] Smajdor, A., 2003, On a multivalued differential problem., Internat. J. Bifur. Chaos Appl. Sci. Engrg., 13, 1877-1882

[38] Tise, I., 2007, Data dependence of the solutions for set differential equations., Carpathian J. Math., 23(1-2), 192-195

[39] A. Tolstonogov, Differential inclusions in a Banach space, Dordrecht, Kluwer Academic Publishers, 2000

[40] Vityuk, A. N., 2003, Differential equations of fractional order with set-valued solutions., Visn. Odes. Derzh. Univ., Ser. Fiz.-Mat. Nauky., $\quad 8(2), 108-112$

[41] Ahmad, B. and Sivasundaram, S., 2008, $\varphi_{0}$-stability of impulsive hybrid setvalued differential equations with delay by perturbing lyapunov functions., Communications in Applied Analysis, 12(2), 137-146

[42] Arsirii, A. V. and Plotnikov, A. V., 2009, Systems of control over set-valued trajectories with terminal quality criterion., Ukr. Math. J., 61(8), 1349-1356

[43] N. D. Phu and T. T. Tung, Multivalued Differential Equations, VNU - HCM City: Publishing House, 2005

[44] Phu, N. D. and Tung, T. T., 2007, Existence of solutions of set control differential equations., J. Sci. Tech. Devel., 10(6), $5-14$

[45] A. V. Plotnikov, "Differential inclusions with Hukuhara derivative and some control problems," Rep. VINITI 26.04.82, 2036-82, 1982

[46] Janiak, T. and Łuczak-Kumorek, E., 2003, Method on partial averaging for functional-differential equations with Hukuhara's derivative. Studia Univ. "Babeş-Bolyai", Math., XLVIII(2), 65-72

[47] Kichmarenko, O. D., 2009, Averaging of differential equations with Hukuhara derivative with maxima., Int. J. Pure Appl. Math., 57(3), 447-457

[48] Kisielewicz, M., 1976, Method of Averaging for Differential Equations with Compact Convex Valued Solutions., Rend. Math., 9(3), 397-408

[49] Plotnikov, V. A. and Kichmarenko, O. D., 2006, Averaging of controlled equations with the Hukuhara derivative., Nonlinear Oscil., 9(3), 365-374 
[50] Plotnikov, V. A. and Rashkov, P. I., Averaging in differential equations with Hukuhara derivative and delay., Funct. Differ. Equ., (8), 371-381

[51] Radstrom, H, 1952, An embeldding theorem for spaces of convex sets., Proc. Amer. Math. Soc., (3), 165-169

[52] Hukuhara, M., 1967, Integration des applications mesurables dont la valeur est un compact convexe., Funkcial. Ekvac., (10), 205-223 